Research Article

\title{
Analysis of Steady State Performance of an Open Loop Drive of a Hydraulic Motor used in Hydrostatic Transmission System
}

\author{
Ajit $\operatorname{Kumar}^{\dot{A}^{*}}$, N. $\operatorname{Kumar}^{\dot{A}}$, K. Dasgupta ${ }^{\dot{A}}$, J. Das ${ }^{\dot{A}}$ and Raj Kumar ${ }^{\dot{\mathrm{B}}}$ \\ ${ }^{\dot{A}}$ Department of Mining Machinery Engineering, ISM, Dhanbad, India \\ ${ }^{\grave{B}}$ HEMM, Shalina Resources, Lubumbashi, Africa
}

Accepted 10 January 2014, Available online 01 February 2014, Special Issue-2, (February 2014)

\begin{abstract}
The speed and torque of the hydraulic motor is controlled by controlling the hydraulic valve pump connected to the hydraulic motor. An electro hydraulic valve coupled to a hydraulic motor is a common method of motor speed control in practice where both load pressure and speed change during operation. In this paper, system equations that include, flow and toque loss term has been established by determining the bent axis motor losses characteristics experimentally. Various experiments have been conducted extensively in the analysis of open loop steady state performance of the hydraulic motor drive system, to determine optimum performance of the system. In investigating the performance open loop drive of the motor, non-dimensional approach is being used to characterize the steady-state performance of the system. This paper denouements the investigation of speed characteristics, efficiency and power transfer of the motor using the non- dimensional analytical approach with respect to the pressure differential across the motor. The characteristics are also validated experimentally.
\end{abstract}

Keywords: hydraulic motor, hydrostatic transmission, steady state performance, non- dimensional approach, speed characteristics.

\section{Introduction}

Hydrostatic Transmission System (HST) mainly consists of hydraulic pump and motor/actuator, which are used to generate, control and transmit the power using pressurized fluid with the help of hydraulic control valves with fast response time. Hydrostatic Transmission System have the capability of being able to control several parameters, such as pressure, speed and position, to a high degree of accuracy and at high power level. This is because it is used to push, pull, regulate or drive virtually all the machines of modern industry. For example, fluid power steers and brakes automobiles, launch spacecraft moves earth harvests crops, mines minerals, military vehicles drives machine tools, elevators, controls airplanes, processes food etc. Liquids provide a very rigid medium for transmitting power and thus can operate under high pressures to provide huge forces and torques to drive loads with utmost accuracy and precision. This system is widely used in mobile equipment also. It is a special case of energy transmission system. In such type of system, the mechanical energy of input drive shaft of the pump is converted to the pressure energy of nearly incompressible working fluid and then re-converted into mechanical energy at the output drive shaft of the bent axis motor with low torque at high speed. There are four types of HST

*Corresponding author: Ajit Kumar

DOI: http://dx.doi.org/10.14741/ijcet/spl.2.2014.75 system based on the type of pumps and motors used, with their corresponding output characteristics:

1) Fixed displacement pump and fixed displacement motor: This system provides constant output power and motor generate constant speed and torque. This is inexpensive and has limited application.

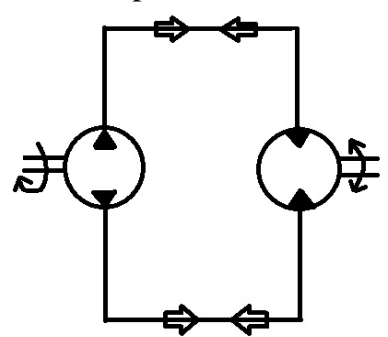

Fig.1

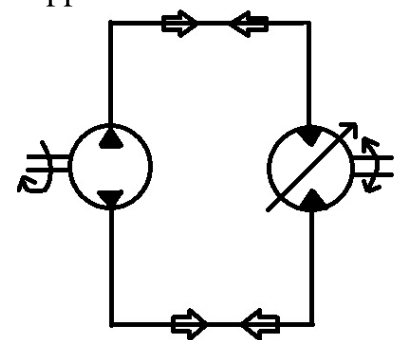

Fig.2
Fig.1 Fixed displacement and fixed displacement motor circuit diagram.

Fig.2 Fixed displacement and variable displacement motor circuit diagram.

2) Fixed displacement pump and variable displacement motor: This is also called a constant power transmission system. This is more expensive than the above type.

3) Variable displacement pump and fixed displacement motor: This is also called constant torque transmission. Power output is varied by 
adjusting the pump displacement with changes with motor speed.

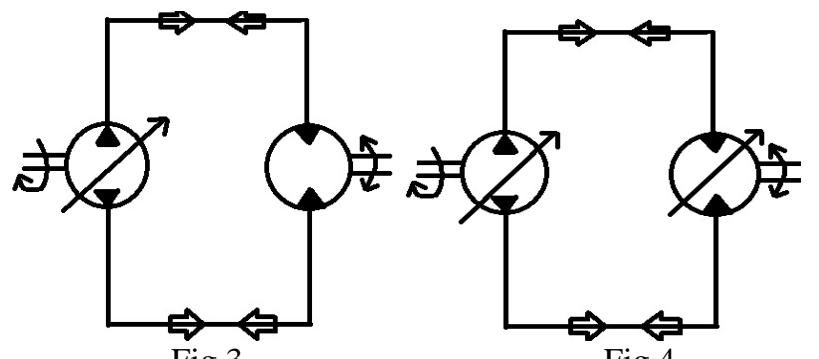

Fig.3 Variable displacement and fixed displacement motor circuit diagram.

Fig.4 Variable displacement and variable displacement motor circuit diagram.

4) Variable displacement pump and variable displacement motor: In this type, varying the displacement of the pump and motor provides a ratio of torque and power. This is the most expensive amongst the other and it is mostly used in heavy earth moving equipments.

HST system, motor speed and torque can also be controlled by controlling the flow supply through the valve placed in between pump and motor. HST has inherent ability to provide driveline flexibility. This unique characteristic allows the cost of the surrounding machine structure to be changed, both by selecting the size and the type of unit and its placement on the machine.

Henke described how the sizing of the components of HST system is done on the basis of the corner horsepower. Numazawa and Okawa have studied the performance of continuously variable transmission on the basis of fuel economy. In their work, it is proposed that such transmission be used with an engine of optimum characteristics. In the performance criteria of positive displacement pumps and fluid motors, Wilson has given the idea for finding out the maximum efficiency of the fluid components based on static analysis. The electrohydraulic servo valve and proportional valves are widely used in industrial applications, such as machine tools, industrial robots, autonomous manufacturing and various actuators in aircraft. They can be applied in closed loop control system. The dynamic analysis of proportional control valve driven hydraulic motor was performed by Ahmed.et.al.. He has analysed the performance limitations of the system due to various mechanical non-linearities. Manring and Luecke has analysed HST system consisting of variable displacement pump and a fixed displacement motor where the system is linearised and the stability range of the system was presented. Watton has analysed hydraulic servo valve controlled motor system based on the liberalized model that is suitable for input of small amplitude. In such a study the detailed dynamics of the servo valve and loading system was considered.

In the presented work, the performance has been investigated of a proportional valve controlled hydraulic motor in an open loop HST system based on the explicit design approach given by Watton.
2. Overview of hydraulic system and physical system considered for analysis

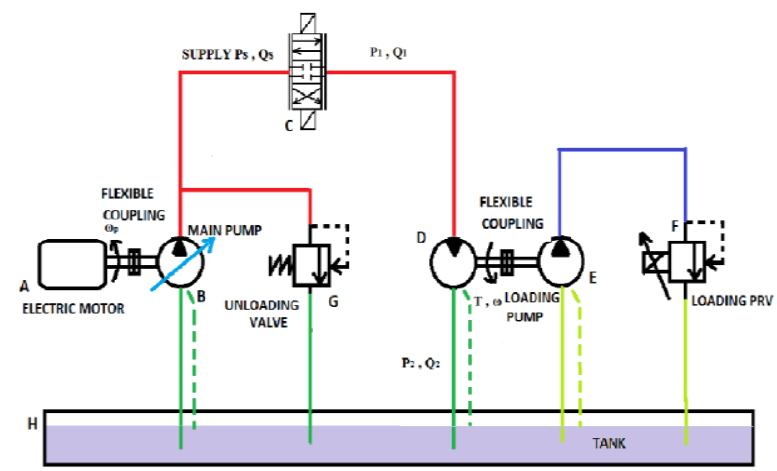

2.1 Hydraulics is broadly classified in two categories:

a) Hydrostatics

b) Hydrodynamics

- Hydrostatics: The study of the confined fluid transmitting power, modifying motion and multiplying forces is called Hydrostatics. In such system, pressure or potential energy of the fluid is more predominant. For example, if fluid is pumped into a closed container at a pressure produced by the hydraulic pump, then according to Pascal's law, the pressure exerts itself equally on all sides of the container - Hydraulic press.

- Hydrodynamics: Systems, in which the kinetic energy of the moving fluid is used, are called Hydrodynamic Systems. In such system, kinetic energy of the fluid is more predominant. Such type of transfer of power is not generally considered as part of fluid power. For example, a jet of water at high velocity impinging on the turbine blades causing the turbine shaft to rotate.

\subsection{Hydrostatic Transmission System [HST]}

In hydrostatic transmission (HST) system the mechanical energy of the input drive shaft of the hydraulic pump is converted to pressure/hydraulic energy in a nearly incompressible working fluid through either open loop or closed loop system and then reconverted into mechanical energy at the output drive shaft of the hydraulic motor through various control line. The system consists of pipes and valves to regulate flow and its direction. It is used to transmit rotating mechanical power from one source to another without the use of mechanical gears.

\subsection{Analysis of the physical system considered for the investigation}

The system considered for open loop drive analysis is shown in figure 5. A fixed displacement pump (B) is driven by the prime mover (A), which is connected to the inlet port of the proportional directional control valve (C). The outlet port of the electro hydraulic proportional valve (C) is connected to the bent axis motor (D) which is loaded by a loading circuit. The loading circuit consists of 
a fixed displacement pump (E), pressure relief valve $(\mathrm{F})$. By controlling proportional pressure relief valve $(F)$, the load torque and speed of the motor (D) are controlled. The load torque $(\mathrm{T})$ and motor speed $(\omega)$ are measured by torque, speed transducer respectively. The test system contains unloading relief valve $(\mathrm{G})$ to protect the system against overloading. Figure 6 shows the physical system considered in the present study.

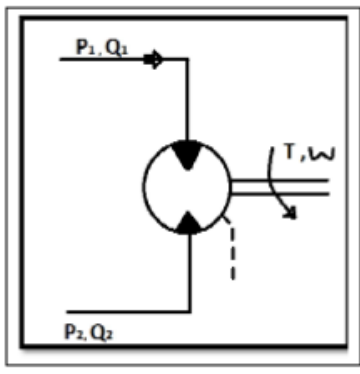

Fig.5 The physical system.

A constant pressure source is maintained to the proportional-valve which drives a fixed displacement bent-axis motor that subsequently drives an identical pump in a loading circuit. The pump loading is controlled through a manually operated pressure relief valve. Some assumptions will be made during this study-

- A constant pressure source of supply to the inlet port of proportional-valve is considered.

- $\quad$ Fluid inertia is neglected.

- Fluid properties have Newtonian characteristics.

- Resistive and capacitive effects are lumped wherever appropriate.

- Losses of the hydrostatic unit are based on the simple and general theory of positive displacement pumps and motors.

- Outlet pressure is assumed to be atmospheric.

- The detail dynamics of the multi-piston assembly, slipper pads etc. of the pump and the motor are not considered.

\section{System analysis and experimental investigation}

\subsection{Steady state characteristics of open loop drive}

The resistances of the proportional-valve ports are modulated depending on the current input to the proportional valve and the pressure difference across the ports. They are considered as modulated flow sources $\left(Q_{1}\right.$ and $\left.Q_{2}\right)$ and expressed as follows:

Inlet flow to the motor $=Q_{1}=k_{f} X_{p v} \sqrt{P_{S}-P_{1}}$

Outlet flow from the motor $=$

$$
Q_{2}=k_{f} X_{p v} \sqrt{P_{2}-P_{\text {sump }}}
$$

But proportional valve spool displacement $\left(X_{p v}\right)$ is directly proportional to the proportional valve amplified current $\left(i_{p v}\right)$. Considering sump pressure $\left(P_{\text {sump }}\right)=$ zero

$$
\begin{aligned}
& Q_{1}=k_{f} X_{p v} \sqrt{P_{S}-P_{1}} \\
& Q_{2}=k_{f} i_{p v} \sqrt{P_{2}}
\end{aligned}
$$

Where, Valve constant $\left(k_{f}\right)=C_{d} \pi d_{P} \sqrt{\frac{1}{\rho}}$

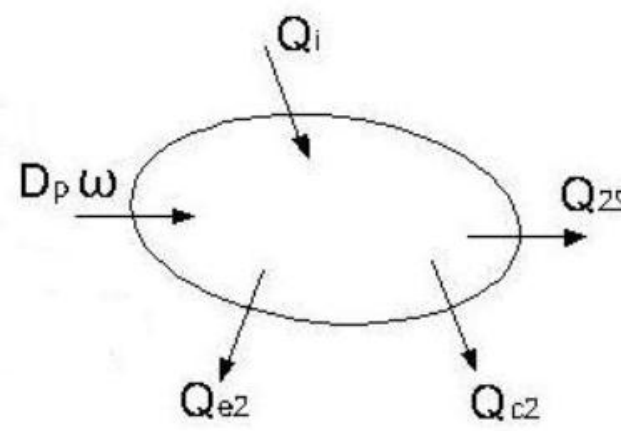

Fig.6 Motor as system.

The pressure difference across the motor $\left(\mathrm{P}_{1^{-}}-\mathrm{P}_{2}\right)$ results in an internal or cross-port leakage $\left(\mathrm{Q}_{\mathrm{i}}\right)$ through the clearance between the rotating barrel inner face and the opposing face. An external leakage flow is $\mathrm{Q}_{\mathrm{e} 1}$ and $\mathrm{Q}_{\mathrm{e} 2}$ represented by the flow from each half of the motor which is taken account during inlet and outlet of the motor flow through the clearance between the rotating barrel outer face and opposing face. Each piston will also have a very small flow loss across its perimeter and from the piston chamber to case, and a much more significant flow loss through the slipper lubrication hole and across the slipper face. These three elements of flow loss are lumped together as a single term external leakage.

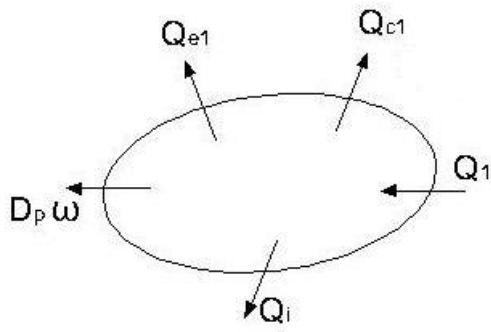

Fig.7 Motor control volume (Inlet \& Outlet)

The additional compressibility losses, $\mathrm{Q}_{\mathrm{c} 1}$ and $\mathrm{Q}_{\mathrm{c} 2}$ are also responsible to reduce the mass flow rate due to pressurization in each line and an apparent flow rate loss due to the dynamic flow mechanism that caused by the fluid inside the motor and casing. The external losses and the compressible losses can't be separated experimentally, and further the equation is reduced to:

$\mathrm{Q}_{\mathrm{e} 1}+\mathrm{Q}_{\mathrm{c} 1}=f_{\mathrm{e} 1}\left(\mathrm{P}_{1}, \omega\right)$

$\mathrm{Q}_{\mathrm{e} 2}-\mathrm{Q}_{\mathrm{c} 2}=f_{\mathrm{e} 2}\left(\mathrm{P}_{2}, \omega\right)$

$\mathrm{Qi}=\mathrm{f}_{\mathrm{i}}\left(\mathrm{P}_{1}, \mathrm{P}_{2}, \omega\right)$

Inlet flow rate to motor $=\mathrm{Q}_{1}=\mathrm{D}_{\mathrm{m}} \omega+\mathrm{Qi}+\mathrm{Q}_{\mathrm{e} 1}+\mathrm{Q}_{\mathrm{c} 1}$

Or, $\mathrm{Q}_{1}=\mathrm{D}_{\mathrm{m}} \omega+\mathrm{f}_{\mathrm{i}}\left(\mathrm{P}_{1}, \mathrm{P}_{2}, \omega\right)+f_{\mathrm{e} 1}\left(\mathrm{P}_{1}, \omega\right)$

Outlet flow rate from motor $=\mathrm{Q}=\mathrm{D}_{\mathrm{m}} \omega+\mathrm{Qi}-\left(\mathrm{Q}_{\mathrm{e} 2}-\mathrm{Q}_{\mathrm{c} 2}\right)$

Or, $\mathrm{Q}_{2}=\mathrm{D}_{\mathrm{m}} \omega+f_{\mathrm{i}}\left(\mathrm{P}_{1}, \mathrm{P}_{2}, \omega\right)-f_{\mathrm{e} 2}\left(P_{2}, \omega\right)$

From equations (5) and (6),

$\mathrm{Q}_{1}-\mathrm{Q}_{2}=f_{\mathrm{e} 1}\left(\mathrm{P}_{1}, \omega\right)+f_{\mathrm{e} 2}\left(P_{2}, \omega\right)$

Since mean flow rate of motor $=\mathrm{Q}_{\mathrm{m}}=\frac{Q_{1}+Q_{2}}{2}$, is linear in nature so flow losses should be linear in nature and depends upon pressure, 
Hence, $f_{\mathrm{e}}(\mathrm{P}, \omega)=\frac{P}{R_{e}}$

Where,

$R_{e}=\frac{P_{1}+P_{2}}{Q_{1}-Q_{2}}$

$\mathrm{Re}=$ coefficient of external flow loss $\left(\mathrm{Nm}^{-2} / \mathrm{m}^{3} \mathrm{~s}^{-1}\right)$

Hence, external flow coefficient $\mathrm{R}_{\mathrm{e}}$ can be expressed as;

$R_{e}=\frac{P_{1}+P_{2}}{Q_{1}-Q_{2}}$

Since the sum of pressure nearly same as supply pressure,

$\mathrm{P}_{1}+\mathrm{P}_{2} \approx \mathrm{P}_{\mathrm{S}}$

Or, $\mathrm{R}_{\mathrm{e}}=\frac{P_{S}}{Q_{1}-Q_{2}}$

It will give the value of external flow loss coefficient $R_{e}$.

Cross port leakage $f_{\mathrm{i}}\left(\mathrm{P}_{1}, \mathrm{P}_{2}, \omega\right)$ is assumed to be linear which is depend upon the pressure difference across the gear motor.

$f_{1}\left(P_{1}, P_{2}, \omega\right)=\frac{P_{1}-P_{2}}{R_{i}}$

Where, $\mathrm{R}_{\mathrm{i}}=$ coefficient of internal flow loss $\left(\mathrm{Nm}^{-2} / \mathrm{m}^{3} \mathrm{~s}^{-1}\right)$ From equations (5) and (6),

$\frac{Q_{1}+Q_{2}}{2}=D_{m} \omega+f_{i}+\frac{f_{e 1}\left(P_{1}, \omega\right)-f_{e 2}\left(P_{2}, \omega\right)}{2}$

From equations (7) and (10),

$\frac{Q_{1}+Q_{2}}{2}=D_{m} \omega+\frac{P_{1}-P_{2}}{R_{i}}+\frac{0.5\left(P_{1}-P_{2}\right)}{R_{e}}$

By considering the mean flow $\left(\mathrm{Q}_{m}\right)$ rate of the fluid through motor,

$Q_{m}=\frac{Q_{1}+Q_{2}}{2}$

From equation (11),

$\frac{1}{R_{i}}=\frac{Q_{m}-D_{m} \omega}{P_{1}-P_{2}}-\frac{0.5}{R_{e}}$

After knowing the value of $R_{e}$ (from equation 8) and $R_{i}$ (from equation 12), flow rate equation (5) and (6) through bent axis hydro-motor can be written as,

$Q_{1}=D_{m} \omega+\frac{P_{1}-P_{2}}{R_{i}}+\frac{P_{1}}{R_{e}}$

And,

$Q_{2}=D_{m} \omega+\frac{P_{1}-P_{2}}{R_{i}}-\frac{P_{2}}{R_{e}}$

Combining equations (13) and (14), the mean flow rate $\left(Q_{\text {mean }}\right)$ may be expressed as;

$Q_{\text {mean }}=\frac{Q_{1}+Q_{2}}{2}=D_{m} \omega_{m}+\frac{P_{1}-P_{2}}{R_{m}}$

Where,

$\frac{1}{R_{m}}=\frac{1}{R_{i}}+\frac{1}{2 R_{e}}$

From equations (13) and (14), the flow difference $Q_{\text {difference }}$ across the motor can be expressed as

$Q_{\text {difference }}=Q_{1}-Q_{2}=\frac{P_{1}-P_{2}}{R}$

Generally for a critically damped position, we assume that $\mathrm{P}_{1}+\mathrm{P}_{2}=\mathrm{KP}_{\mathrm{S}}$ and if there is load or restriction in line then, $\mathrm{P}_{1}-\mathrm{P}_{2}=\mathrm{P}_{\text {load }}$. Hence, equation (3) can be written as:

$Q_{1}=k_{f} i_{p v} \sqrt{\frac{P_{s}-P_{\text {load }}}{2}}$

Description for non-dimensional flow equation reference value is taken at no load conditions.

For no load conditions $\mathrm{P}_{1}=\mathrm{P}_{2}$ as $\mathrm{P}_{\text {load }}=0$,
$Q_{\text {reference }}=k_{f} i_{p v} \sqrt{\frac{P_{S}}{2}}=\mathrm{D}_{\mathrm{m}} \omega(0)$

Dividing equation (3) by $Q_{\text {reference }}$,

$Q_{1}=k_{f} i_{p v}-\sqrt{P_{S}-P_{1}}$

$\frac{Q_{1}}{Q_{\text {reference }}}=\frac{k_{f} i_{p v}-\sqrt{P_{S}-P_{1}}}{k_{f} i_{p v} \sqrt{\frac{P_{S}}{2}}}$

$=\sqrt{\frac{2 P_{S}-2 P_{1}}{P_{S}}}$

$=\sqrt{2-\frac{P_{1}+P_{2}}{P_{S}}-\frac{P_{1}-P_{2}}{P_{S}}}$

$=\sqrt{2-\frac{K P_{S}}{P_{S}}-\frac{P_{\text {load }}}{P_{S}}}$

Or,

$\overline{Q_{1}}=\sqrt{2-K-P_{\text {load }}}$

From equation (16), $Q_{2}$ can be expressed as,

$Q_{2}=Q_{1}-\frac{P_{1}+P_{2}}{R_{e}}=Q_{1}-\frac{K P_{S}}{R_{e}}$

Dividing this equation by $Q_{\text {reference }}=k_{f} i_{p v} \sqrt{\frac{P_{S}}{2}}$

$\frac{Q_{2}}{Q_{\text {reference }}}=\frac{Q_{1}}{Q_{\text {reference }}}-\frac{K P_{S}}{R_{e} k_{f} i_{p v} \sqrt{P_{S} / 2}}$

Or,

$\frac{Q_{2}}{Q_{\text {reference }}}=\overline{Q_{1}}-\frac{K P_{S}}{R_{e} k_{f} i_{p v} \sqrt{P_{S} / 2}}$

Or,

$\overline{Q_{2}}=\overline{Q_{1}}-L=\sqrt{2-K-P_{\text {load }}}-L$

Where,

$\frac{K P_{s}}{R_{e} k_{f} i_{p v} \sqrt{P_{s} / 2}}=\frac{K P_{s}}{R_{e} D_{m} \omega(0)}=L=$ Constant

Or,

$\overline{Q_{2}}=\sqrt{2-K-P_{\text {load }}}$

Non dimensional mean flow rate can be expressed as,

$\overline{Q_{m}}=\frac{Q_{1}+Q_{2}}{2}=\frac{Q_{1}+Q_{2}}{2 D_{m} \omega(0)}$

Or,

$\overline{Q_{m}}=\frac{1}{2}\left(2 \sqrt{2-K-\overline{P_{\text {load }}}}-L\right)$

Or,

$\overline{Q_{m}}=\left(\sqrt{2-K-\overline{P_{\text {load }}}}\right)$

It is proven graphically and experimentally, by comparing the practical and ideal cases for different feasible values of $\mathrm{K}$ that the error in assuming $\left(\mathrm{P}_{1}+\mathrm{P}_{2}\right) \cong \mathrm{P}_{\mathrm{S}}$ i.e. $\mathrm{K}=1$ for flow rate calculation can be assumed to be negligible for practical load pressure, depending at the speed being considered.

Considering the above system equations, a mean flow approximation may be used with sufficient accuracy to calculate system performance. From above equation flow rate may me expressed as

$Q_{1}=D_{m} \omega(0)\left(\sqrt{1-\bar{P}_{\text {load }}}\right)$ 


$$
\begin{aligned}
& Q_{2}=D_{m} \omega(0)\left[\left(\sqrt{1-\bar{P}_{\text {load }}}\right)-L\right] \\
& Q_{\text {mean }}=D_{m} \omega(0)\left[\left(\sqrt{1-\bar{P}_{\text {load }}}\right)-\frac{L}{2}\right]
\end{aligned}
$$

3.2 Non dimensional approach for speed of motor From equation (20), it can be written as,

$$
\begin{aligned}
& \frac{Q_{1}+Q_{2}}{2}=\overline{Q_{m}} \times D_{m} \omega(0) \\
& \text { Or, } \\
& \begin{aligned}
D_{m} \omega+\frac{P_{1}-P_{2}}{R_{i}} & +\frac{P_{1}-P_{2}}{2 R_{e}} \\
& =D_{m} \omega(0) \times\left[\left(\sqrt{1-\bar{P}_{\text {load }}}\right)-\frac{L}{2}\right]
\end{aligned}
\end{aligned}
$$

Or

$$
\frac{D_{m} \omega+\frac{P_{1}-P_{2}}{R_{i}}+\frac{P_{1}-P_{2}}{2 R_{e}}}{D_{m} \omega(0)}=\left[\left(\sqrt{1-\bar{P}_{\text {load }}}\right)-\frac{L}{2}\right]
$$

Or,

$\frac{\omega}{\omega(0)}=\left[\left(\sqrt{1-\bar{P}_{\text {load }}}\right)-\frac{L}{2}-\frac{P_{1}-P_{2}}{D_{m} \omega(0)}\left(\frac{1}{R_{i}}+\frac{1}{2 R_{e}}\right)\right]$

Or,

$\bar{\omega}=\sqrt{1-\bar{P}_{\text {load }}}-\frac{P_{S}}{2 R_{e} D_{m} \omega(0)}-\frac{P_{L}}{R_{m} D_{m} \omega(0)}$

Or,

$$
\bar{\omega}=\sqrt{1-\bar{P}_{\text {load }}}-\frac{P_{S}}{2 R_{e} D_{m} \omega(0)}\left(\frac{R_{m}}{2 R_{e}}+\frac{P_{L}}{P_{S}}\right)
$$

Or,

$\bar{\omega}=\sqrt{1-\bar{P}_{\text {load }}}-\propto\left(\frac{R_{m}}{2 R_{e}}+\frac{P_{L}}{P_{S}}\right)$

Or,

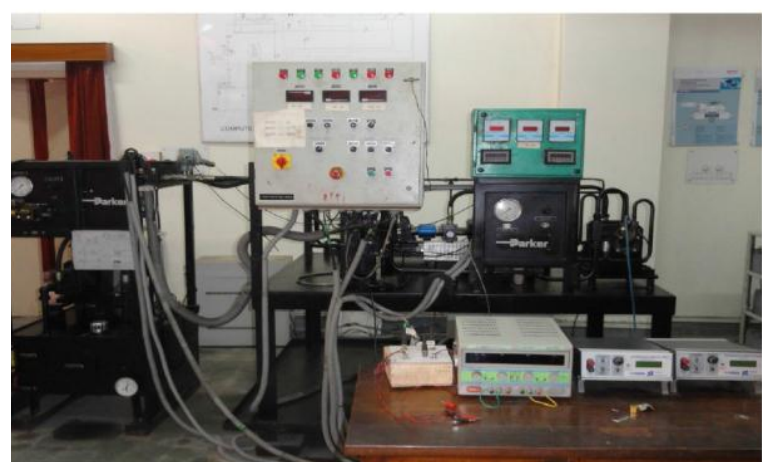

Fig 8 Experimental setup

Or,

$$
\bar{\omega}=\sqrt{1-\bar{P}_{\text {load }}}-\propto\left(\frac{R_{m}}{2 R_{e}}+\bar{P}_{\text {load }}\right)
$$

$\bar{\omega}=\sqrt{1-\bar{P}_{\text {load }}}-\propto\left(\frac{R_{m}}{2 R_{e}}+\bar{P}_{\text {load }}\right)$

$\omega=\omega(0)\left\{\sqrt{1-\bar{P}_{\text {load }}}-\propto\left(\frac{R_{m}}{2 R_{e}}+\bar{P}_{\text {load }}\right)\right\}$

$\omega=f\left(\bar{P}_{\text {load }}\right)=f 1\left(\bar{P}_{\text {load }}\right)$

Where,

$\frac{P_{S}}{R_{m} D_{m} \omega(0)}=\alpha=$ constant
By solving equation (21), as given above, the simulation results of motor speed $(\omega)$ with respect to the non dimensional load pressure $\overline{\mathrm{P}}_{\text {load }}$ is given in Figure 8 .

It is clear from the graph that with the decrease in the ratio of pressure difference at the inlet $\&$ outlet ports and the system pressure, the hydro motor speed varies and it increases taking a linear graph with the decrease in the ratio of pressure difference and the system pressure.I

\subsection{Experimental analysis}

The experiment was conducted using the test setup shown in fig. 10. The computer controlled hydraulic motor setup is a proportional valve controlled system. A brief description of the test setup is given below:

Fig.9 Front view of test set up

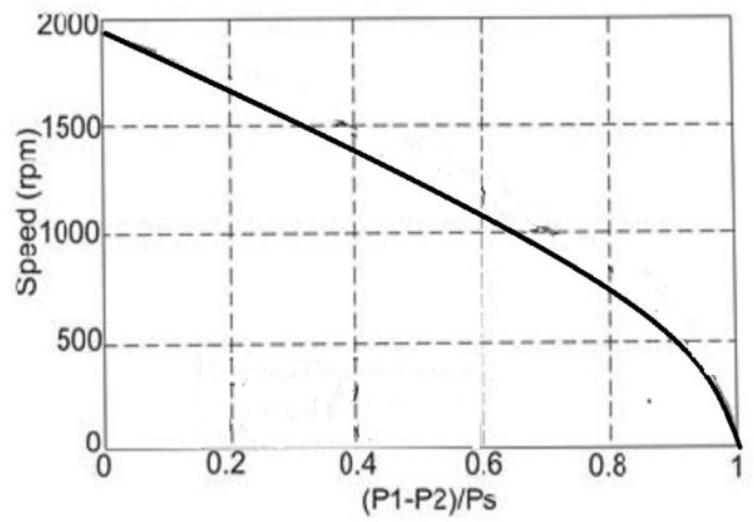

Fig.9 The theoretical speed characteristics of proportional valve controlled bent -axis hydro motor at no - load speed of $2000 \mathrm{rpm}$

A variable displacement pump is driven by electric motor (A.C induction motor). This pump supplies pressurized hydraulic fluid to hydraulic motor through check valve, pressure line filter and directional control valve (either proportional or servo). Check valve doesn't allow fluid to flow in reverse direction from DC valve to tank. Hence it always maintains some fluid in fluid line. DC valve (proportional) and DC valve (servo) are four ways; open centered and closed centered valves respectively. In proportional or servo controlled valves, spool movement and hence output flow are proportional to input current signal. Input command signal to this valve can be varied by microprocessor controller.

In this way this hydraulic system is operated to determine performance characteristics of open loop drive of a Hydraulic motor.

- $\quad$ Speed, power and efficiency at no load motor speed of $2246 \mathrm{rpm}$

The characteristics curves of speed, power transfer and efficiency versus load pressure at no load condition having motor speed of $2246 \mathrm{rpm}$ have been drawn by taking experimental data from the test set up. The curves depicts the nature of the critical parameters viz. speed, power and efficiency of the hydraulic system at a motor speed of $2246 \mathrm{rpm}$. The derived equations are applicable in determining the motor performance for its wide range of operation. The various characteristics curves are shown below in fig. 10, 11 and 12 . 


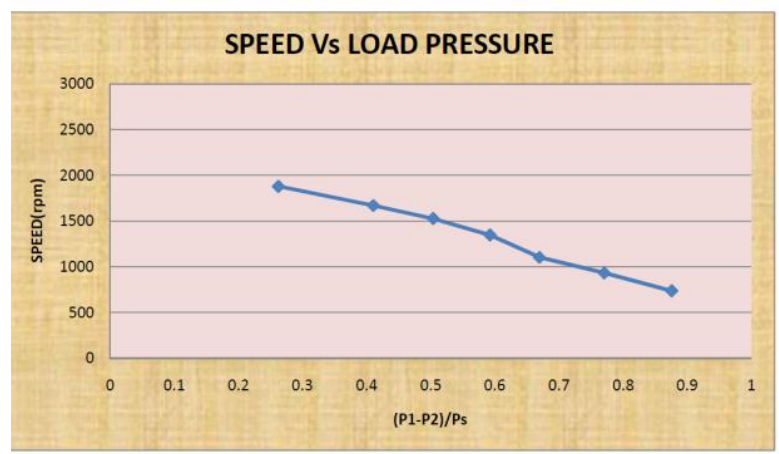

Fig.10 Speed Vs Load pressure at no load motor speed of $2246 \mathrm{rpm}$

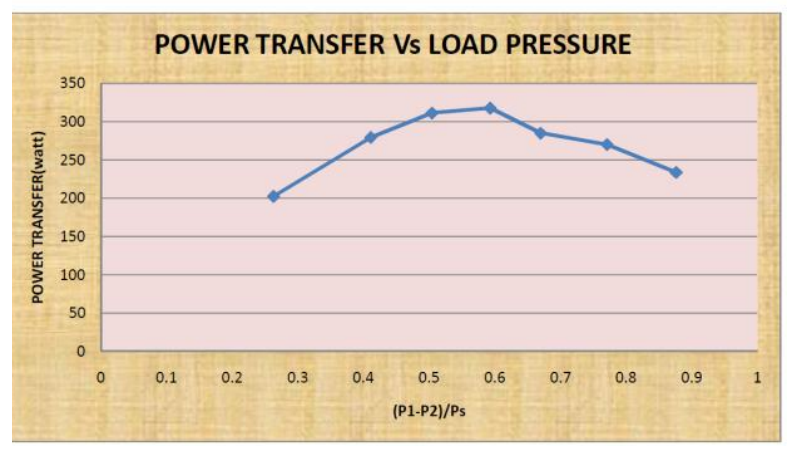

Fig.11 Power transfer Vs Load pressure at no load motor speed of $2246 \mathrm{rpm}$

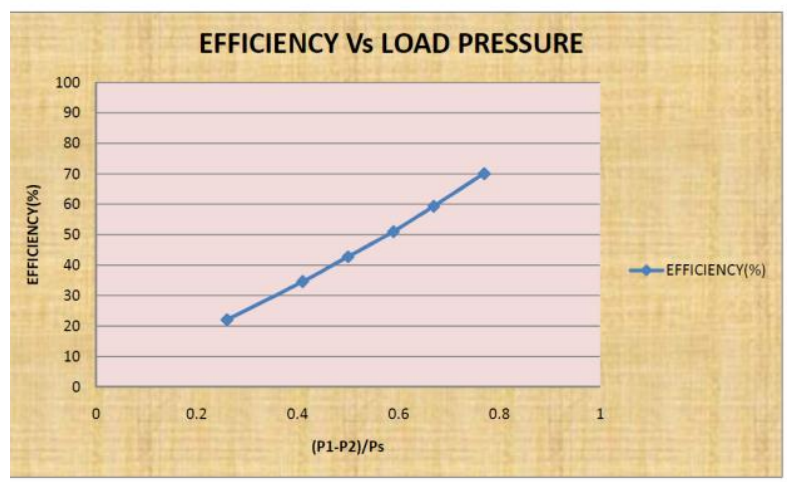

Fig.12 Efficiency Vs Load pressure at no load motor speed of $2246 \mathrm{rpm}$

\section{Conclusions}

The deduction that the ideal flow equation is sufficiently accurate for evaluating motor speed, drive efficiency and motor power transfer in practice is particularly significant and useful, since it lead to ability to drive a directly set of explicit design equation. The flow across bent axis hydro motor has linear dependency at different speed with pressure difference across the motor. The system design equations allow direct determination of the conditions for maximum efficiency. These derived design equations become more accurate in determining the power transfer particularly for higher no-load speeds. For open loop operation, the maximum power transfer to the load occurs at a load pressure $\mathrm{P}_{1}-\mathrm{P}_{2} \cong(2 / 3) \mathrm{P}_{\mathrm{S}}$ and it is independent of the motor leakage losses. The derived equations are applicable in determining the motor performance for its wide range of operation. Dynamic performance of bent axis hydro motor can be studied as a future work of this research.

\section{Acknowledgement}

An astronomical number of teachers, engineering lovers and classmates have made valuable and profound suggestions which we have incorporated in this work. It is not possible for us to acknowledge all of them individually. We take this opportunity to express my gratitude to them. Before, we express my thanks; we seek pardon for any omissions which is purely unintentional. With all humility we wish to express my deep sense of gratitude to Prof. (HOD) K. Dasgupta, Department of Mining Machinery Engineering, Indian School of Mines, Dhanbad, for his vigilant, for providing all types of lab facilities desiderated to carry out this project work, for sharing his knowledge \& experience with me thereby adding another brick to the wall in making and caring guidance at every stage of this research. We would like to thank my colleagues, especially Abhishek Kumar and Akanksha Sinha, for their support in our study and experimental work. We would like to acknowledge the help of all other non teaching staff and officials of the Department of Mining Machinery Engineering. We would like to thank all the authors, who have contributed to make this work possible. Last but not the least, we would like to thank our parents and family members for their support and encouragements in our current project work.

\section{References}

J. Watton, (1989), Closed-loop design of an electro-hydraulic motor drive using open-loop steady state characteristics, The Journal of Fluid Control and Fluidics, Quarterly 20 (1), pp. 7-30.

H.E. Merritt, (1967), Hydraulic Control Systems, Wiley, New York.

H. Murrenhoff, (1969), Systematic approach to the control of hydrostatic drives, Journal of Systems and Control, ImechE, Part-I 213 (15), pp. 333-347.

J. Watton, (1986), The stability of electro-hydraulic servomotor systems with transmission lines and non-linear motor friction effects. Part A: System modeling, The Journal of Fluid Control Including Fluidics, Quarterly 16 (2-3), pp. 118-136.

K. Dasgupta, (2000), Analysis of hydrostatic transmission system using low-speed-high-torque motor, Mechanism and Machine Theory, vol. 35, pp. 1481-1499.

R.W.Henke, (1982), Hydraulic transmission system, Hydraulic and Pneumatic Power,

Numazawa \& Okawa, (1981), An analysis study of vehicle performance \& fuel economy potential of continuous variable transmission system, Hydraulic and Pneumatic Power. W.E. Wilson, (1949), Performance criteria for positive displacement pump \& fluid motor, ASME, USA.

J. Watton, (1989), Closed-loop design of an electro-hydraulic motor drive using open-loop steady state characteristics, The Journal of Fluid Control and Fluidics, Quarterly 20 (1), pp. 7-30.

S. R. Majumdar, Oil hydraulic system principle and maintenance.

Michael J Pinches, John G Ashby, Oil hydraulics.

John Watton, Fundamentals of Fluid Power Control, Cambridge University Press. 